\title{
LA CALIDAD DE LA EDUCACIÓN: LA CUARTA VÍA PARA EL DESARROLLO ECONÓMICO
}

\author{
Rafael Macherone*
}




\section{RESUMEN}

Apoyándose en la evidencia de diversos estudios, el artículo enfatiza la importancia de ver la calidad de la educación como el verdadero motor del desarrollo económico. Se advierte que las sociedades que no siguen políticas acertadas en materias educacionales corren serios riesgos de perder competitividad, sumergirse indefinidamente en el subdesarrollo y acrecentar la brecha con los países desarrollados. Entre las medidas encaminadas a promover la mejora de la calidad de los sistemas educativos destacan: la orientación hacia los resultados en el sentido de intensificar los procesos de evaluación de alumnos, profesores y establecimientos educacionales; el refuerzo de las oportunidades de calidad para todos, debido a las grandes diferencias de rendimiento académico y laboral que producen las actuales desigualdades en materia de calidad; la mayor flexibilidad y apertura de los currículum con el objeto de desarrollar las competencias que exige la nueva era del conocimiento y, finalmente, las políticas orientadas a elevar la condición social del profesorado.

\section{ABSTRACT}

Based on the evidence from several studies, the paper emphasizes the importance of looking to the quality of education as the true engine of economic development. It points out that societies that fail to follow adequate policies in educational matters run serious risks of losing competitiveness, submerging themselves indefinitely in underdevelopment and widening the gap with developed countries. Some noteworthy measures seeking to promote the quality of the educational systems include: resultsoriented systems in the sense of enhancing the assessment of students, teachers and educational centers; reinforcing the opportunity of quality for all, due to the major differences in academic and work performance resulting from current disparities in terms of quality; greater flexibility and opening up of the curricula in order to develop the competencies required by the new era of learning and, finally, policies aimed at raising the social status of teachers. 


\section{LA CALIDAD DE LA EDUCACIÓN: LA CUARTA VÍA PARA EL DESARROLLO ECONÓMICO}

Hace dos o tres décadas pocos dudaban de que la educación fuera un elemento crucial para el logro del desarrollo económico. Un estudio del economista Sebastián Piñera (1980) afirmaba que la educación no sólo constituía un objetivo en sí mismo, sino que además cumplía un doble papel. Por una parte, era uno de los campos más efectivos de intervención pública en la tarea de redistribuir ingresos y erradicar la pobreza y, por otra, representaba una de las formas de inversión más eficaces para acelerar el proceso de desarrollo.

No obstante, lo que concretamente se hacía en materia educacional en Chile y en el mundo, dos o tres décadas atrás, era bastante primario. Si bien se avanzaba en el desafío de aumentar la tasa de escolaridad y se debatía acerca de reformas educacionales, nada es comparable con el desarrollo y la importancia que ha adquirido el tema de la educación en los últimos 10 años.

Hoy la preocupación por la educación es un imperativo ineludible. En la actual "era del conocimiento" las sociedades que no emprenden políticas acertadas en materias educacionales corren serios riesgos de perder competitividad, sumergirse indefinidamente en el subdesarrollo y acrecentar la brecha con los países desarrollados.

Diversos estudios han demostrado la alta rentabilidad privada de invertir en educación. Wirt (1999)* y otros muestran que en EE.UU., en 1996, un individuo de sexo masculino, entre 25 y 34 años, con grado de Bachellor ${ }^{1}$ o superior, percibía un ingreso 54\% mayor que aquel que sólo había completado su enseñanza media (high school).

\footnotetext{
* Véase bibliografía final.

1. Grado otorgado por las instituciones de educación superior norteamericanas (colleges o universidades) que equivale aproximadamente al grado académico de Licenciado que se otorga en nuestro país.
} 
Por otra parte, aquellos que sólo tenían entre 9 y 11 años de escolaridad, vale decir una enseñanza secundaria incompleta, percibían un ingreso 31\% inferior al de aquellos que la habían completado. En el caso de las mujeres, el estudio muestra que los años de escolaridad tienen un efecto aún más importante sobre los ingresos. Las mujeres entre 25 y 34 años, con un grado de Bachellor o superior, obtenían un ingreso $88 \%$ superior al de aquellas que sólo habían completado la enseñanza media.

La mayor atención actual al tema educacional está presente en prácticamente todas las latitudes. Así, países como España, preocupados por haber vivido buena parte del siglo XX en inferiores condiciones que la mayoría de sus vecinos europeos, hoy quieren tomar la delantera en esta materia.

El proyecto de Ley Orgánica de Calidad de la Educación (LOCE) de este país señala que "La educación se encuentra hoy en el centro de los desafíos y de las oportunidades de las sociedades del siglo XXI y nunca ha sido tan evidente que calidad y equidad, desarrollo económico y cohesión social, no son elementos contrapuestos, sino objetivos ineludibles, a la vez que complementarios, del avance de nuestras sociedades".

Entre las medidas encaminadas a promover la mejora de la calidad de los sistemas educativos destacan: la orientación hacia los resultados, el refuerzo de las oportunidades de calidad para todos, la mayor flexibilidad y apertura de los currículum y las políticas orientadas a elevar la condición social del profesorado.

\section{ORIENTACIÓN HACIA RESULTADOS}

La orientación hacia los resultados conduce a la intensificación de los procesos de evaluación de alumnos, profesores y establecimientos educacionales.

En materia de evaluación de alumnos los avances de los últimos años han sido significativos. Muchos países están sometiendo a los alumnos que cursan el cuarto y octavo año de enseñanza primaria a 
mediciones nacionales del nivel de aprendizaje que alcanzan los estudiantes de distintas escuelas. También han proliferado las pruebas y estudios de medición de aprendizaje que tienen por objeto realizar comparaciones internacionales.

Uno de estos estudios es el TIMSS², que permitió hacer comparaciones internacionales en el aprendizaje de las matemáticas y las ciencias en estudiantes que cursan el cuarto y octavo grado. Particularmente favorecidos en esta medición resultaron los alumnos del sistema educacional japonés, quienes se ubicaron consistentemente en los primeros lugares del ranking internacional. En ciencias alcanzaron el segundo lugar en cuarto grado y el tercero en octavo, mientras que en matemáticas ocuparon el tercer lugar tanto en cuarto como en octavo grado ${ }^{3}$.

Wu (1999)* argumentó que esta privilegiada posición no se alcanzó, como algunos observadores creen, debido a los altos estándares que se han impuesto en matemáticas y ciencias las escuelas japonesas o al gran número de horas destinadas a estas materias, sino a métodos educacionales expresamente diseñados para motivar a los alumnos a aprender. El interés por aprender se consigue en las escuelas japonesas con la participación activa de los estudiantes en las aulas. En lugar de dictar una lección convencional, los profesores solicitan a un estudiante particular que presente a la clase su solución a un determinado problema; luego le pide al resto de la clase que evalúe la solución. De esta forma, los alumnos cooperan en el proceso de aprendizaje, no ven a sus pares como adversarios, sienten que sus ideas son respetadas y adquieren más confianza en sus habilidades para aprender.

Para esta tarea se requieren profesores dispuestos a cambiar los hábitos de instrucción tradicional, hecho que no es trivial, puesto que significa moverlos desde un lugar cómodo a un lugar de menor seguridad y requiere una fuerte inversión inicial en términos de horas de dedicación.

2. Third International Mathematics and Science Study.

3. La muestra del estudio incluyó 26 países en cuarto grado y 41 países en octavo grado.

* Véase bibliografía final. 
Unido a lo anterior está el hecho que expresa Perlmutter (1987)*: "Cada día es más evidente que no es posible enseñar a los alumnos todo lo que deberían aprender. Consecuentemente, un nuevo abordaje se impone: la enseñanza debe mostrar a los alumnos cómo aprender, cómo ser independientes, autoorientados e instrumentalizados para enfrentar un ambiente en constante cambio".

\section{OPORTUNIDAD DE CALIDAD PARA TODOS}

Es sabido que en ninguna parte del mundo los alumnos tienen la oportunidad de recibir un nivel de educación de calidad pareja y que las diferencias en la calidad de la educación recibida marcan fuertemente las posibilidades de éxito en la educación superior y posteriormente en el ámbito laboral.

El trabajo de Horn y Kajaku (2002)* concluye que la rigurosidad del currículum de la enseñanza secundaria en EE.UU. tiene una alta asociación con el prestigio de la universidad o college al que ingresan los estudiantes. Mientras más alta la rigurosidad del currículum, más oportunidades tienen los alumnos de ser aceptados en universidades más selectivas ${ }^{4}$. En el período 1995-1996, el 71\% de los estudiantes que completaron un currículum riguroso fue aceptado en universidades selectivas, comparado con un $40 \%$ que cursó un currículum de nivel medio y un 32\% que completó sólo un currículum básico ${ }^{5}$.

El trabajo de Horn y Kajaku concluye también que el nivel de rigurosidad del currículum en la enseñanza secundaria estadounidense está fuertemente relacionado con las probabilidades de permanencia en la universidad y con la obtención del grado de Bachellor. Tres años después de su ingreso, independientemente de que los estudiantes se mantuvieran en la misma institución a la que ingresaron o se cambiaran a otra universidad, un $87 \%$ de los alumnos que habían cursado un

* Véase bibliografía final.

4. La selectividad está medida por los puntajes que obtienen los alumnos en las pruebas de selección a la educación superior (SAT).

5. Cabe señalar que del total de alumnos que ingresó a la universidad en el período 1995-96, un $19 \%$ cursó currículum rigurosos, un 50\% currículum de nivel medio y un $31 \%$ de currículum básicos. 
currículum riguroso continuaban su carrera hacia la obtención del Bachellor -que se otorga al concluir un plan de estudios de cuarto año-, contra un $71 \%$ de quienes tenían un currículum de nivel medio y un $62 \%$ de los que cursaron un currículum básico.

\section{MAYOR FLEXIBILIDAD Y APERTURA}

Para atender a las nuevas exigencias y retos de la sociedad moderna, la educación debe preparar a los estudiantes para vivir en un mundo global y cambiante. Para ello se requiere que adquieran destrezas tales como la capacidad de comunicarse, trabajar en equipo y tener la facilidad de adoptar nuevas tecnologías.

En este plano, el estudio de lenguas extranjeras tiene un gran valor si se quiere agregar una dimensión internacional a los currículum. Adelman (1994)* señala que en EE.UU., en los últimos años, se ha dado especial énfasis al estudio de la cultura no-occidental, a la historia, la geografía y la política. En los ochentas el principal argumento de este esfuerzo de integración era la creciente competitividad de la economía global. En los noventas el argumento se basa en la necesidad de que los estudiantes entiendan una sociedad y un mundo multicultural.

También es necesario desarrollar planes de estudios que se ajusten a las nuevas realidades. Al respecto, hace un año y medio el Presidente Ricardo Lagos señaló: "Resulta paradójico que en un mundo cada vez más demandante de competencias nuevas en el campo de las telecomunicaciones, en la ciencia, la tecnología, el diseño y el arte, nuestros jóvenes sigan enfrentando el viejo dilema de elegir prácticamente las mismas líneas de formación de pregrado. Cuando vemos cuáles fueron las disciplinas de Bello, y cuáles son las disciplinas de hoy ipor Dios!, hay más similitud con el Chile de 1842 y sus disciplinas que con las que podemos intuir se van a necesitar en el siglo XXI, en el cual ya nos encontramos"6.

* Véase bibliografía final.

6. Extracto del discurso pronunciado por el Presidente Ricardo Lagos en la inauguración del año académico 2000 de la Universidad de Chile. 


\section{LA IMPORTANCIA DEL PROFESORADO}

Las políticas orientadas a elevar la condición social del profesorado y atraer hacia esta profesión a los mejores estudiantes son un elemento decisivo para lograr la eficacia y la eficiencia de los sistemas de educación.

En este terreno los profesores japoneses presentan ventajas con respecto a sus pares de otros países. Ellos muestran un fuerte deseo de mejorar su rendimiento aprendiendo de sus colegas, manteniendo una fluida interacción acerca de los currículum y acerca de cómo mejorar las técnicas pedagógicas (Wu, 1999)*.

Mejorar la calidad de los profesores constituye uno de los factores más importantes para el logro de una apropiada educación y formación de los estudiantes. A pesar del alto reconocimiento de este factor, Ingersoll (1999)* comenta que resulta sorprendente que se trate de uno de los tópicos menos comprendidos en educación.

Hay poco consenso en cómo definir la calidad de la performance de los profesores y es empíricamente muy difícil medirla. Hay consenso universal con respecto a la importancia de los programas de entrenamiento para profesores, pero, en general, estos programas dejan mucho que desear.

En esta línea tienen gran importancia los esfuerzos que puede hacer el Estado para motivar a los estudiantes hacia la profesión docente. Jennings (1999)* destaca que en EE.UU. el Acta Federal de Educación Superior se modificó para incluir incentivos financieros tanto a los egresados de los colleges que se dedicaran a la docencia como a las instituciones de educación superior que destinaran esfuerzos a la formación de mejores profesores. En Chile se están comenzando a hacer esfuerzos similares.

La tarea no es fácil, más aún cuando el rápido crecimiento del número de alumnos no ha ido aparejado con el incremento en la cantidad de nuevos profesores. Esto ha conducido, en muchos casos, a una reducción de los estándares a la hora de reclutar nuevos académicos.

* Véase bibliografía final. 
Panorama sobre la formación universitaria en Chile.

\section{REFERENCIAS Bibliográficas y Notas}

Adelman, Clifford, "What Employers expect of College Graduate: International Knowledge and Second Language Skills"; en Educational Research Report, EE.UU., julio 1994.

Ministerio de Educación de España, Proyecto de Ley Orgánica de Calidad de la Educación.

Fernández, M. J. y González, A., "Desarrollo y situación actual de los estudios de eficacia escolar"; en Relieve, Volumen 3, Número1, España, 1997.

Fitzgerald, Robert A., "College Quality and Earnings of Recent College Graduate"; en Education Statistics Quarterly, EE.UU., otoño 2001.

Horn, Laura y Lawrence, Kojaku, K., "High School Academic Curriculum and the Persistence Path Through College: Persistence and Transfer Behavior of Undergraduates 3 Years After Entering 4Years Institutions"; en Education Statistics Quarterly, EE.UU., otoño 2002.

Ingersoll, Richard M., "Understanding the Problem of Teacher Quality in American Schools"; en Education Statistics Quarterly, EE.UU., primavera 1999.

Jennings, John F., "Better Policies Leading to Improved Teaching"; en Education Statistics Quarterly, EE.UU., primavera 1999.

Lagos, Ricardo, Discurso con motivo de la inauguración del año académico 2000 de la Universidad de Chile; en Diario Estrategia, Santiago, Chile, 23 de marzo de 2000, p. 29.

Perlmutter, M., "Dynamique d'un groupe d'apprendissage", WHO EDUC, Canadá, 1987.

Piñera, Sebastián, "Orientaciones para una reforma al sector educacional chileno"; en Cuadernos de Economía No 50, Pontificia Universidad Católica de Chile, Santiago, Chile, abril 1980. 
Supovitz, J., y Poglinco, S. M., "Instructional Leadership in a Standardsbased Reform"; en Consortium for Policy Research in Education, EE.UU., diciembre 2001.

Wirt, J. y otros, "Annual Earnings of Young Adults by Educational Attainment"; en Education Statistics Quarterly, otoño, 1999, EE.UU.

Wu, Angela, "The Japanese Education System: A Case Study Summary and Analysis"; en Research Today, EE.UU., enero 1999. 